\title{
AUTORREGULACIÓN DEL APRENDIZAJE Y COMPRENSIÓN DE TEXTOS: ESTUDIO DE INTERVENCIÓN
}

\section{SELF-REGULATED LEARNING AND READING COMPREHENSION: AN INTERVENTION STUDY}

\author{
Daniel Trías \\ Universidad Católica del Uruguay, Uruguay \\ Juan Antonio Huertas \\ Universidad Autónoma de Madrid, España
}

\begin{abstract}
Resumen: Evaluar el impacto de la enseñanza de la autorregulación del aprendizaje sobre la comprensión de textos y estrategias de autorregulación constituye el propósito del presente estudio de intervención. Participaron 81 estudiantes de bachillerato de Montevideo, Uruguay. En este estudio cuasi-experimental con grupo control, se asignó como condición experimental una metodología dirigida a la enseñanza de la autorregulación en contexto de aula, a cargo del docente y en nueve horas de clase. Se evaluó colectivamente la comprensión de textos, antes y después, utilizando cuestionarios. Finalizada la intervención, se utilizó una tarea de pensamiento en voz alta para evaluar estrategias de autorregulación. Los alumnos del grupo experimental alcanzaron niveles significativamente superiores de comprensión de textos. En ese grupo se beneficiaron especialmente los alumnos de rendimiento menor. Los sujetos del grupo experimental exhiben conductas de autorregulación. Se discuten los alcances de la metodología utilizada y sus posibilidades de utilización en la enseñanza.
\end{abstract}

Palabras clave: autorregulación del aprendizaje; comprensión de textos; motivación.

\begin{abstract}
The purpose of this intervention study is to assess the impact of teaching self-regulatory learning on reading comprehension and self-regulatory strategies. Eighty-one high school students from the city of Montevideo participated in the study. A quasi-experimental design was used; the experimental condition involved teaching a self-regulation strategy in the classroom over nine hours of regular classroom time. The control group was taught the same material by the same teacher with no self-regulation training intervention. Reading comprehension was assessed before and after the intervention, using questionnaires. Upon completion of the intervention, a think-aloud procedure was used in order to evaluate students' use of self-regulation strategies. Subjects in the experimental group achieved significantly higher levels of reading comprehension. In addition, those students in the experimental group with lower academic performance especially benefited. In the experimental group, subjects exhibited self-regulation behaviors. The scope of the utilized methodology and its potential for use in educational contexts are discussed.
\end{abstract}

Keywords: self-regulated learning; reading comprehension; motivation.

\section{INTRODUCCIÓN}

La brecha entre el mejoramiento de la práctica educativa y los conocimientos teóricos que se acumulan, continúa desafiando particularmente a la investigación en psicología educacional y anima el trabajo que aquí se presenta.

Vamos a exponer los resultados de un pequeño estudio de intervención realizado en contexto de aula con alumnos de bachillerato.

En la asignatura de Filosofía se propuso a un docente utilizar una metodología dirigida a la enseñanza de la autorregulación del aprendizaje y se evaluó el impacto de dicha intervención sobre la comprensión de textos y la actividad de autorregulación que los estudiantes realizan.

Este trabajo se alinea junto con aquellos que buscan formas eficaces de favorecer la autorregulación del aprendizaje en contextos de aula, desde las premisas teóricas que la investigación ha venido aportando y puesto a prueba, fundamentalmente, en contextos de tipo experimental y muy controlados.

Correspondencia: Daniel Trías, Departamento de Psicología del Desarrollo y Educación. Facultad de Psicología. Universidad Católica del Uruguay. Correo electrónico: gtrias@ucu.edu.uy. 
La autorregulación del aprendizaje ha resultado de sumo interés para la investigación en las últimas décadas y ha sido abordada desde diferentes paradigmas (Paris y Paris, 2001; Zimmerman, 2008). Desde la práctica educativa y de cara a los desafíos que una sociedad de cambios tan rápidos plantea, ha cobrado relevancia la necesidad de que los estudiantes perfecciones sus competencias relacionadas con el aprendizaje autónomo. Los conceptos de "aprender a aprender", "estrategias de aprendizaje", "habilidades metacognitivas" hacen evidente tal necesidad (Pozo, 2008) y se vinculan directamente a los estudios sobre aprendizaje autorregulado.

El presupuesto básico de estos postulados y de los diversos estudios que se han realizado es considerar que quienes autorregulan su aprendizaje están comprometidos activamente en los procesos de construcción del significado y adaptan sistémicamente sus pensamientos, emociones y acciones al servicio del logro de sus metas en función de un contexto dado (Boekaerts y Corno, 2005; Zimmerman 2000).

Boekaerts y Corno (2005) brindan un panorama sobre la investigación de la autorregulación en las últimas décadas y las variaciones que ha sufrido este concepto. A su juicio, entre los años 70 y 80 la autorregulación del aprendizaje fue concebida como una capacidad general, que las personas poseían o no, independientemente del contexto. La investigación en autorregulación en la década del 90 se caracterizó por el esfuerzo por atrapar los componentes motivacionales y volitivos, a la vez por la búsqueda de modelos que den cuenta del papel del contexto en la autorregulación. La presente década se caracteriza por la inclusión decidida de los factores sociales y emocionales en los modelos de autorregulación, a la vez se concibe como un proceso que se desarrolla y se pone en juego en función de la situación.

La evolución de las concepciones de autorregulación ha estado acompañada de cambios en los instrumentos de evaluación. Concebida como una capacidad general, se desarrollaron cuestionarios y entrevistas estructuradas que buscaban atrapar las regularidades en la actuación del estudiante. En un segundo período se propusieron instrumentos que fueran más sensibles al contexto de dominio específico.

En la actualidad, es marcada la necesidad de triangular instrumentos, y a la vez evaluar la autorregulación situada, aquí y ahora, en acción para aproximarse más al conocimiento del proceso de autorregulación (Boekaerts y Corno, 2005). La tarea de pensamiento en Voz alta constituye un ejemplo vigente de ese tipo de evaluación (Braten y Samuelstuen, 2004; Zimmerman 2008).

No sólo han cambiado las maneras de concebir y evaluar la autorregulación del aprendizaje, también lo han hecho las formas de intervención dirigidas a la enseñanza de la autorregulación. El interés por los experimentos en contexto de laboratorio se ha desplazado a los estudios de intervención sobre enseñanza de la autorregulación en contextos de aula, con mayor validez ecológica, que pueden aportar al entendimiento de cómo el contexto incide en el sistema de autorregulación.

Paris y Paris (2001) enfatizan la necesidad de identificar explícitamente en las aulas cómo se operativizan las concepciones de aprendizaje autorregulado. Destacan fundamentalmente dos razones para ello: la necesidad de sintetizar la cuantiosa investigación acumulada y vincularla con la práctica educativa; la posibilidad de poner a un mayor número de alumnos en condiciones más ventajosas con métodos que los docentes puedan utilizar y ajustar efectivamente en sus clases.

Estudios con universitarios (Nietfeld, Cao y Osborne, 2005; Onwuegbuzie, Slate y Schwartz, 2001) evidencian que el desarrollo de la autorregulación no es consecuencia ni de la edad, ni del nivel educativo. Al tiempo que abonan la premisa que sostiene que sin enseñanza difícilmente se desarrollarán niveles crecientes de autorregulación del aprendizaje. Una de las interrogantes que mantienen vigencia en la investigación es cómo los alumnos llegan a autorregular su aprendizaje (Paris y Paris, 2001).

Dicho de otro modo, la pregunta que resuena en los contextos de enseñanza-aprendizaje es cómo transferir saberes, habilidades y poder, para que sea el alumno quien controla su propio aprendizaje (Zimmerman, 2008).

Teniendo en cuenta la especificidad de dominio encontramos estudios de intervención en ámbitos muy diferentes como: comprensión de textos (Mason, 2004), producción de textos (Torrance, Hidalgo, \& García, 2007; Guasch, \& Castelló, 2002), resolución de problemas matemáticos (Fuchs, Fuchs, Prentice \& cols., 2003; Butler, Beckingham, \& Novak, 2005) y ciencias naturales (Sungur \& Takkaya; 2006). 
Este trabajo ha hecho foco en la enseñanza de la autorregulación en Filosofía, centrándose en los procesos de autorregulación implicados en la comprensión de textos filosóficos. Buena parte de las diferencias individuales en la comprensión de textos son explicadas por la actividad de monitoreo que los lectores realizan (Eme, Puustinen, \& Coutelet, 2006). Quienes alcanzan niveles más altos de monitoreo autorregulan su estudio y obtienen mejores resultados en las pruebas académicas (Thiede, Anderson, \& Therriault, 2003).

Entre los escasos estudios de intervención en aula y con estudiantes de secundaria, se encuentra la evaluación de la eficacia de un programa de enseñanza de estrategias de lectura (Alfassi, 2004). El programa combinaba enseñanza directa y recíproca en el contexto de aula y formaba parte del currículum de los estudiantes. Sus resultados avalan la hipótesis que la enseñanza de las estrategias de comprensión con la metodología combinada redunda en un incremento de los resultados en tareas de comprensión de textos. Las mejoras observadas se producen a partir de una intervención que responde a la heterogeneidad de un salón de clases, sin alterar significativamente los contenidos del curso.

Son poco frecuentes los estudios en contexto de aula que evalúen la eficacia de las intervenciones educativas en el largo plazo y en las habilidades significativas para el éxito académico. Esta ausencia de investigación posiblemente se vincule a las dificultades que impone investigar científicamente en educación. También a la distancia que se genera entre la investigación básica y la intervención educativa. La relación entre ambas debería ser complementaria, pues una comprensión científica más profunda debería aportar prácticas efectivas para mejorar la educación (Pintrich, 2003).

El propósito de este trabajo es evaluar el impacto de una metodología para la enseñanza de la autorregulación en contexto de aula en la comprensión de textos y los procesos de autorregulación implicados en la lectura de un texto.

En relación a la comprensión de textos, las hipótesis que se manejan son que: a) los alumnos que reciban enseñanza explicita de autorregulación obtendrán mejores resultados en comprensión de textos; b) que los alumnos de peor rendimiento que participen del grupo experimental incrementarán sus niveles de comprensión luego de la intervención.

Considerando los procesos de autorregulación implicados en la lectura de textos, se hipotetiza que: a) los alumnos del grupo experimental evidenciarán conductas de autorregulación al leer un texto de la asignatura; b) a mayor rendimiento académico los alumnos exhibirán mayor proporción de conductas de autorregulación al leer un texto filosófico.

\section{MÉTODO}

\section{PARTICIPANTES}

La muestra la constituyeron 81 estudiantes que cursaban el penúltimo año de Bachillerato en Uruguay. Contaban con 16 años cumplidos, siendo un $54,3 \%$ de sexo masculino y un 45,7 $\%$ de sexo femenino. Participaron de la intervención en clase y de todas las evaluaciones 68 alumnos. De los alumnos restantes disponemos de datos parciales, pues no completaron la totalidad de instancias de evaluación realizadas en horas de clase.

La intervención se desarrolló en un colegio de nivel socioeconómico medio-alto de Montevideo, Uruguay. Con autorización explícita de la Dirección de dicha institución se seleccionaron intencionalmente dos grupos de clase, en los que el mismo docente dictaba la asignatura de Filosofía, con uno se formó el grupo de control y con otro el experimental. Se informó a los alumnos que estaban participando de un programa para el mejoramiento de la enseñanza de la asignatura, y las actividades propuestas formaban parte del curso regular.

\section{INSTRUMENTOS}

Guión para la enseñanza de la autorregulación en la lectura de textos en Filosofía: se ha elaborado siguiendo las sugerencias metodológicas para la instrucción metacognitiva realizadas por Mateos (2001). Los aspectos destacados en el guión eran: i) jerarquizar el proceso presentado como el que él emplea, pues le resulta útil; ii) activar conocimientos previos; iii) conformar redes semánticas; iv) sortear dificultades que los textos presentan; v) necesidad que cada alumno asuma un rol activo buscando su propia forma.

Tarea de pensamiento en voz alta: evalúa la autorregulación que se lleva a cabo durante la realización de la tarea (Cerdán 2007; Cerdán y Vidal-Abarca, 2008; Zimmerman, 2008). 
Se propuso a cada sujeto la lectura de un texto y que a la vez explicitará en voz alta todo aquello que fuera pensadominetras comprendía el texto.

Luego se realizaron preguntas vinculadas al contenido y a los procedimientos que el alumno sugeriría utilizar frente a un pasaje similar.

El texto se seleccionó entre los sugeridos en el programa y aún no trabajados en clase.

Se decidió que fuera el docente quien planteara la entrevista, en vistas a ganar en validez ecológica y educativa al recibir el estudiante retroalimentación de la persona competente.

Pruebas de comprensión de textos: se diseñaron dos cuestionarios, siguiendo la propuesta metodológica de Alonso-Tapia y Carriedo (2002). Dos textos distintos se seleccionaron entre los que, a juicio de los docentes de Filosofía, los alumnos deberían leer en el curso. Cada texto fue utilizado en la evaluación previamente a ser trabajado en el curso, uno en el pre-test y otro en el post-test. Con el pasaje delante debían responder a dos preguntas abiertas que evaluaban la construcción de la macroestructura. Fueron corregidas por dos jueces, con un nivel de acuerdo de .80. Las discrepancias fueron resueltas por consenso. Ya sin el texto delante, respondían a ocho preguntas cerradas sobre el contenido que variaban su puntuación entre $0,1,2$, a menor y mayor corrección. Para ambos cuestionarios se realizaron estudios de consistencia interna.

Se obtuvo un valor Alpha de Cronbach de .71, para el primer cuestionario. Mientras en el segundo cuestionario se obtuvo un Alpha de .49; si bien el valor es bajo, dado el número de estudiantes y la validez demostrada de este tipo de pruebas decidimos seguir utilizando.

\section{DISEÑO}

Se trata de un estudio cuasi-experimental de intervención con grupo control.

La variable independiente fue el tipo de enseñanza, asignando al grupo experimental una metodología dirigida a la enseñanza de la autorregulación. Antes y después se midió la variable dependiente: comprensión de textos. Finalizada la intervención se midió la autorregulación del aprendizaje.

El rendimiento académico (en tres niveles, considerando la calificación final en la asignatura) fue considerado como variable agrupadora.

\section{PROCEDIMIENTO}

Obtenida la autorización correspondiente de parte de la Dirección de la institución, se elaboró el guión para enseñar autorregulación. Se explicitaron y seleccionaron algunos procedimientos que el docente, como experto en Filosofía, utiliza para autorregular su aprendizaje en textos y que enseñaría a sus alumnos. El guión fue formulado en palabras del docente. Se utilizaron instancias de modelado y de moldeamiento de acuerdo a una secuencia planificada por el docente.

Inicialmente se evaluó a todos los participantes de forma colectiva, utilizando el primer cuestionario de comprensión de textos.

La intervención en aula, a cargo del docente, transcurrió durante tres semanas (nueve horas de clase). En el grupo experimental el docente trabajaba en base al guión para la enseñanza de la autorregulación cuando introducía los textos previstos en la disciplina. La secuencia implicaba el pasaje gradual de instancias de enseñanza directa al trabajo autónomo de los alumnos. Mientras en el grupo control destacaba los conocimientos previos al presentar un texto. Las clases fueron grabadas magnetofónicamente. Paralelamente se mantenía una reunión semanal con el docente, y se recibían sus registros de planificación.

Finalizada la intervención se seleccionaron seis alumnos del grupo experimental (al azar, en bloques conformados por nivel de rendimiento). El propio docente les propuso la tarea de pensamiento en voz alta. De ese modo dispuso de información que le permitió ajustar su planificación.

En la semana final del curso se administraron colectivamente nuevos cuestionarios de comprensión de textos y se registraron las calificaciones finales de cada alumno en la asignatura. Se volvió a entrevistar al docente una vez finalizado el curso académico y nuevamente al terminar el año siguiente, de manera de contar con una medida de transferencia.

\section{RESULTADOS}

Se presentarán las comparaciones entre el grupo experimental y control, así como los tres niveles de rendimiento, en comprensión de textos y procesos de autorregulación. Se consideró un nivel de confianza mayor al 95\% para todas las pruebas estadísticas, realizadas utilizando el programa SPSS. 


\section{Comprensión de textos}

Una primera hipótesis consideraba que los alumnos que han recibido enseñanza explicita de autorregulación obtendrían mejores resultados en comprensión de textos. A los efectos de las realizar las comparaciones en comprensión se consideró el promedio expresado en porcentaje de los ítems 9 (“¿Cómo titularías el texto?") y 10 ("Intenta explicarle a alguien que no ha leído el texto las ideas que presenta. Utilizando tus palabras, escribe un párrafo con ese objetivo"). Estos ítems mostraron ser empíricamente los más representativos y tener la correlación más alta en los análisis factoriales exploratorios de cada cuestionario (método de los Componentes Principales y la rotación Varimax). Ambos constituyen un buen indicador de la construcción del significado global del texto, se respondían teniendo el texto delante e interrogaban de la misma forma.

En la Tabla 1 pueden observarse las medias y desviaciones estándar de cada grupo en ambas evaluaciones. Se presentan expresados en porcentaje, pues se trata de ítems que tenían distinto rango de variación en cada cuestionario. En el primer cuestionario, tal como era esperable ambos grupos no se diferenciaron significativamente en sus niveles de comprensión de textos, comparados con la prueba de Mann-Whitney. En tanto, en la evaluación final, existe un rendimiento superior del grupo experimental. Mediante la prueba de Mann-Whitney se obtiene una significación asintótica de .027. En relación a la primera hipótesis, se evidencia un rendimiento sensiblemente superior del grupo experimental al explicitar en forma escrita la comprensión de las ideas principales del texto.

Para calibrar la evolución de cada grupo en el tiempo se utilizó la prueba de rangos con signos de Wilcoxson. En el segundo cuestionario el grupo experimental al explicitar por escrito las ideas principales del texto alcanza resultados similares a los del primer cuestionario. En tanto, el grupo control tiene rendimientos significativamente decrecientes, con un valor de significación asintótica de .027. Ante un texto de mayor dificultad, los alumnos del grupo experimental mantienen su rendimiento y se desempeñan mejor que los sujetos del grupo control, quienes reflejan menores posibilidades de explicitar las ideas centrales del texto. Cabe destacar que las evaluaciones se realizaron con textos diferentes y correspondían a contenidos de la asignatura.

A juicio del docente, el texto propuesto en la segunda evaluación resulta más difícil. Parece necesario analizar quiénes se benefician de la intervención dirigida a la enseñanza de la autorregulación. Una segunda hipótesis es que los alumnos de peor rendimiento que participaron del grupo experimental incrementarían sus niveles de comprensión. En la Tabla 2 se observan las medias y desviaciones estándar en los cuestionarios de comprensión agrupados según sus calificaciones finales (rendimiento bajo, intermedio y alto). Se utilizó la prueba de rangos con signos de Wilcoxon para la comparación de los resultados en comprensión antes y después considerando los niveles de rendimiento.

\begin{tabular}{|c|c|c|c|c|}
\hline & \multicolumn{2}{|c|}{$\begin{array}{c}\text { CUESTIONARIO 1 } \\
\text { PROMEDIO ÍTEMS 9 Y 10 } \\
\text { EN PORCENTAJE }\end{array}$} & \multicolumn{2}{c|}{$\begin{array}{c}\text { CUESTIONARIO 2 } \\
\text { PROMEDIO ÍTEMS 9 Y 10 } \\
\text { EN PORCENTAJE }\end{array}$} \\
\hline & $\bar{X}$ & $\mathrm{~S}$ & $\bar{X}$ & $\mathrm{~S}$ \\
\hline Experimental & 47,5 & 36,0 & 47,7 & 27,3 \\
\hline Control & 45,2 & 35,2 & 34,0 & 24,2 \\
\hline
\end{tabular}

Tabla 1. Comprensión de textos. Medias y desvíos estándar.

\begin{tabular}{|c|c|c|c|c|c|c|c|}
\hline & & \multicolumn{2}{|c|}{$\begin{array}{c}\text { RENDIMENTO } \\
\text { BAJO }\end{array}$} & \multicolumn{2}{|c|}{ RENDIMENTOINIERNEDIO } & \multicolumn{2}{|c|}{$\begin{array}{c}\text { RENDIMENTO } \\
\text { ALTO }\end{array}$} \\
\hline & & Cuest. 1 & Cuest. 2 & Cuest. 1 & Cuest. 2 & Cuest. 1 & Cuest. 2 \\
\hline \multirow[b]{2}{*}{ EXPERIMENTAL } & $\overline{\bar{X}}$ & 11,1 & 51,2 & 41,7 & 45,8 & 58,3 & 52,4 \\
\hline & s & 4,8 & 35,8 & 35,0 & 26,7 & 36,1 & 25,4 \\
\hline \multirow[b]{2}{*}{ CONIROL } & $\bar{X}$ & 13,3 & 14,3 & 38,9 & 29,3 & 67,9 & 40,6 \\
\hline & s & 25,4 & 11,0 & 34,6 & 23,5 & 30,0 & 21,4 \\
\hline \multirow[t]{2}{*}{ TODOS } & $\bar{X}$ & 13,6 & 22,6 & 39,8 & 33,0 & 60,5 & 45,3 \\
\hline & s & 19,1 & 26,9 & 33,4 & 27,4 & 33,4 & 27,1 \\
\hline
\end{tabular}

Tabla 2. Comprensión de textos. Medias y desviaciones estándar, por grupos y niveles de rendimiento. 
Parece necesario analizar quiénes se benefician de la intervención dirigida a la enseñanza de la autorregulación. Una segunda hipótesis es que los alumnos de peor rendimiento que participaron del grupo experimental incrementarían sus niveles de comprensión. En la Tabla 2 se observan las medias y desviaciones estándar en los cuestionarios de comprensión agrupados según sus calificaciones finales (rendimiento bajo, intermedio y alto). Se utilizó la prueba de rangos con signos de Wilcoxon para la comparación de los resultados en comprensión antes y después considerando los niveles de rendimiento.

En el post-test, ante un texto más difícil, los alumnos del grupo experimental muestran niveles de comprensión más homogéneos. Los alumnos de rendimiento bajo (3 sujetos) aumentaron sus niveles de comprensión, beneficiándose de la intervención. Alcanzaron una media similar a la de los alumnos de alto rendimiento. Sin embargo, este incremento no resulta estadísticamente significativo, dado el reducido número de alumnos y la variabilidad de sus resultados. Los alumnos de rendimiento intermedio y alto no varían significativamente sus niveles de comprensión (ver Gráfico 1).

A diferencia del grupo experimental, los niveles de comprensión de los alumnos del grupo control son heterogéneos (ver Gráfico 1).

Los alumnos de bajo rendimiento (5 sujetos) mantienen muy bajos niveles de comprensión en ambas evaluaciones. Tampoco los alumnos de rendimiento intermedio muestran diferencias.
En cambio, los alumnos de alto rendimiento en el grupo control muestran un descenso estadísticamente significativo al explicitar las ideas principales del texto; con una significación asintótica de 0,019. No logran sortear los obstáculos que el segundo texto les ofrecía.

\section{Autorregulación del aprendizaje}

Nuestras últimas hipótesis aventuraban que los alumnos del grupo experimental evidenciarían conductas de autorregulación al leer un texto de la asignatura. Además, sus estrategias de autorregulación serían mejores en los estudiantes conforme obtuviesen mejor rendimiento académico. En general, los indicadores que pudimos obtener van en esta línea.

Se evaluó a seis sujetos del grupo experimental con tareas de pensamiento en voz alta. No fue posible recuperar el registro fílmico de uno de los alumnos de alto rendimiento, por lo que se ha excluido del análisis. Los registros de esta tarea fueron analizados por un observador externo, usando categorías pre-determinadas. Se contabilizaron las frecuencias absolutas de las conductas observadas y se calcularon los porcentajes de cada conducta en relación al total de las conductas observadas en cada entrevista, tal como puede verse en la Tabla 3.

Los alumnos evidencian diversas formas de resolución de la tarea de pensamiento en voz alta y todos exhiben conductas de autorregulación. Estas conductas comunes a todos los sujetos evaluados son: realizar comentarios pertinentes sobre el texto, manifestaciones de entendimiento y releer espontáneamente.

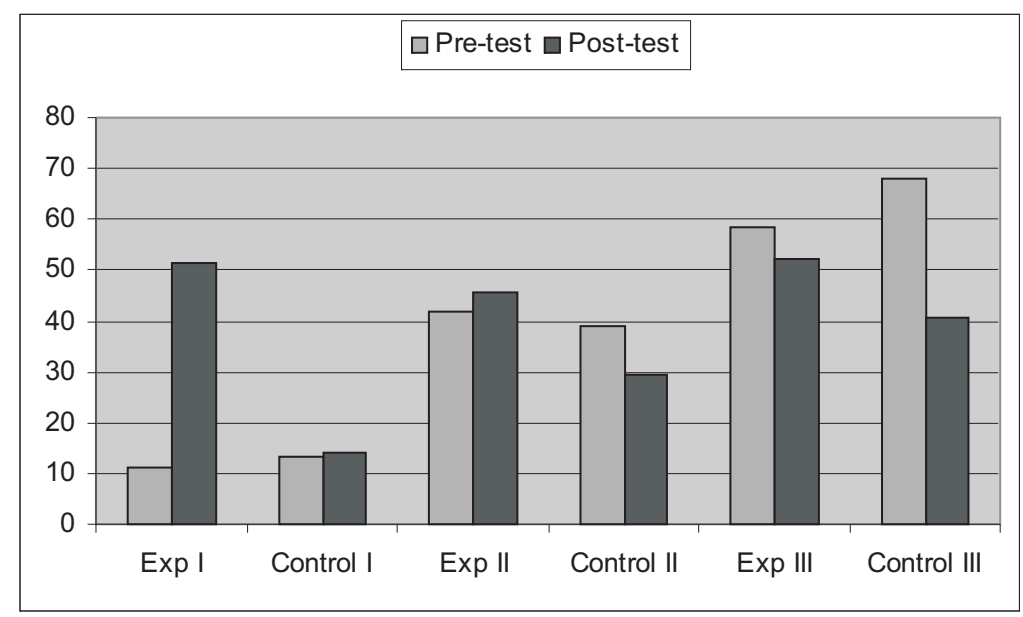

Gráfico 1. Comparación de niveles de comprensión antes y después, según condición experimental y agrupados por niveles de rendimiento. 


\begin{tabular}{|c|c|c|c|c|c|c|c|c|}
\hline & \multicolumn{5}{|c|}{$\begin{array}{c}\text { Conductas observadas en cada sujeto, } \\
\text { porcentaje sobre el total. }\end{array}$} & \multirow[b]{2}{*}{$\bar{X}$} & \multirow[b]{2}{*}{$\mathrm{s}$} \\
\hline & & $\begin{array}{l}\text { Suj.1 } \\
\text { Bajo }\end{array}$ & $\begin{array}{l}\text { Suj. } 2 \\
\text { Bajo }\end{array}$ & $\begin{array}{l}\text { Suj. } 3 \\
\text { Medio } \\
\end{array}$ & $\begin{array}{l}\text { Suj. } 4 \\
\text { Medio }\end{array}$ & $\begin{array}{l}\text { Suj. } 5 \\
\text { Alto }\end{array}$ & & \\
\hline \multirow{9}{*}{ 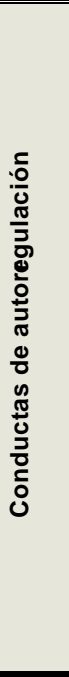 } & $\begin{array}{c}\text { Realiza } \\
\text { comentarios } \\
\text { pertinentes del }\end{array}$ & 10,0 & 8,8 & $18,5^{\star}$ & 17,6 & 12,0 & 13,4 & 4,4 \\
\hline & $\begin{array}{l}\text { Expresa } \\
\text { autopreguntas }\end{array}$ & 0,0 & 11,7 & 11,1 & $29,4^{*}$ & 8,0 & 12,0 & 10,8 \\
\hline & $\begin{array}{l}\text { Manifiesta que ha } \\
\text { entendido }\end{array}$ & 10,0 & 2,9 & 11,1 & $23,5^{\star}$ & 12,0 & 11,9 & 7,4 \\
\hline & Subraya & 0,0 & 0,0 & 3,7 & 0,0 & $36,0^{*}$ & 7,9 & 15,8 \\
\hline & $\begin{array}{c}\text { Relee } \\
\text { espontáneamente }\end{array}$ & 5,0 & 5,9 & $14,8^{*}$ & 5,9 & 8,0 & 7,9 & 4,0 \\
\hline & $\begin{array}{l}\text { Vuelve y se detiene } \\
\text { en alguna palabra } \\
\text { o expresión }\end{array}$ & $15,0^{*}$ & 8,8 & 7,4 & 0,0 & 4,0 & 7,0 & 5,6 \\
\hline & $\begin{array}{c}\text { Utiliza el título y/o } \\
\text { refiere a } \\
\text { conocimientos } \\
\text { previos }\end{array}$ & 0,0 & 2,9 & $11,1^{*}$ & 5,9 & 4,0 & 4,8 & 4,1 \\
\hline & $\begin{array}{l}\text { Expresa criterios } \\
\text { que utiliza }\end{array}$ & 0,0 & 0,0 & 0,0 & 0,0 & 8,0 & 1,6 & -- \\
\hline & $\begin{array}{l}\text { Evalúa extensión } \\
\text { del texto }\end{array}$ & $5,0^{*}$ & 2,9 & 0,0 & 0,0 & 0,0 & 1,6 & 2,3 \\
\hline \multirow{2}{*}{ 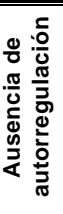 } & $\begin{array}{l}\text { Manifiesta falta de } \\
\text { comprensión }\end{array}$ & $45,0^{*}$ & 29,4 & 18,5 & 17,6 & 8,0 & 23,7 & 14,1 \\
\hline & $\begin{array}{l}\text { No avanza ante } \\
\text { dificultad de } \\
\text { vocabulario }\end{array}$ & 10,0 & $26,5^{*}$ & 3,7 & 0,0 & 0,0 & 8,0 & 11,1 \\
\hline \multicolumn{2}{|c|}{$\begin{array}{l}\text { Porcentaje de conductas de } \\
\text { autorregulación en total de } \\
\text { observaciones }\end{array}$} & 45,0 & 44,1 & 77,8 & 82,3 & 92,0 & 68,2 & 22,3 \\
\hline
\end{tabular}

Tabla 3. Autorregulación. Tarea de pensamiento en voz alta.

Evaluar la extensión del texto es visible solo en los alumnos de bajo rendimiento. Mientras que expresar los criterios que utiliza durante la tarea es un comportamiento visible solo en el sujeto de alto rendimiento.

En los alumnos de bajo rendimiento se observan los menores niveles de autorregulación, correspondiendo a un 45,0 y $44,2 \%$ de conductas de autorregulación. Mayoritariamente las conductas observadas en los alumnos de este nivel corresponden a manifestaciones de inseguridad o de dificultad. El porcentaje de conductas de autorregulación aumenta a 77,8 y $82,3 \%$ en los alumnos de rendimiento intermedio.

Quien exhibe mayor porcentaje de conductas de autorregulación, un 92\%, es alumno de alto rendimiento.

A medida que aumenta el nivel de rendimiento académico, se observan en mayor porcentaje las conductas de autorregulación implicadas en la lectura del texto.

\section{DISCUSIÓN Y CONCLUSIONES}

En relación a la primera hipótesis, se observó un efecto positivo de la enseñanza de la autorregulación en la comprensión de textos. En el post-test fue significativamente superior el desempeño del grupo experimental. Aquellos a quienes se enseñó explícitamente autorregulación del aprendizaje, están en mejores condiciones de reflejar el significado global del texto al asignar un título y resumir en un párrafo las ideas principales del mismo.

Las diferencias a favor del grupo experimental se hacen visibles en las tareas que exigen mayor complejidad cognitiva (dar cuenta de la macro-estructura) y en textos más complejos. Ante un texto de dificultad mayor, el grupo experimental mantiene niveles similares de comprensión a los alcanzados en el primer texto. Por el contrario, el grupo control muestra menores niveles de comprensión. Estas diferencias al reflejar por escrito las ideas principales del texto se vinculan a que los alumnos 
del grupo experimental alcanzan niveles superiores de monitoreo y han ganado en estrategias de autorregulación que les permiten sortear las dificultades del texto (Eme, Puustinen, \& Coutelet, 2006; Thiede, Anderson, \& Therriault, 2003).

Los alumnos de bajo rendimiento del grupo experimental muestran un crecimiento muy importante en su media de comprensión.

En la segunda evaluación alcanzan niveles similares a los alumnos de rendimiento intermedio y alto de su grupo. Mientras los alumnos de bajo rendimiento del grupo control mantienen muy bajos niveles de comprensión. La intervención dirigida a la enseñanza de los procesos de autorregulación beneficia sobre todo a alumnos de bajo rendimiento. Esto realza la utilidad de la enseñanza explícita de estrategias metacognitivas para la comprensión de textos (Roeschl-Heils, Schneider \& Kraayenoord, 2003) en aquellos estudiantes que no han podido conseguir todavía estrategias de supervisión de la comprensión.

Como era lógico pensar, se observa que los alumnos a los cuales se ha enseñado explícitamente a autorregular el aprendizaje exhiben también conductas de autorregulación en la tarea de pensamiento en voz alta. Este dato nos sirve para validar nuevo el método de pensamiento en voz alta al converger nuestros resultados en esta tarea con los obtenidos en los cuestionarios que aquí pasamos y que evidencian que a mayores niveles de rendimiento, mayor presencia de conductas de autorregulación (Kitsantas, 2002).

A su vez, cada alumno evidencia formas diferentes y propias de autorregulación. La autorregulación no necesariamente depende de la edad o el nivel educativo, los niveles de autorregulación que se observan son resultado de los aprendizajes explícitos e implícitos de cada persona (Nietfeld, Cao \& Osborne, 2005; Onwuegbuzie, Slate \& Schwartz, 2001). En nuestro caso ha sido la enseñanza explícita de los procesos de autorregulación lo que ha impactado sobre los niveles de comprensión de textos. La literatura especializada (ver Paris y Paris, 2001) ha mostrado recientemente las dificultades que existen para conseguir que lo que parece que funciona en enseñanza de la autorregulación en entornos controlados se pueda transferir a las aulas reales, a la enseñanza.
En este sentido nuestros datos muestran que los efectos observados en comprensión de textos y autorregulación del aprendizaje conseguidos, se vinculan a una metodología de intervención relativamente sencilla y económica, que puede desarrollarse en contexto de aula sin modificar de forma grande la enseñanza y sin complicar mucho el escenario formativo. El eje central de la misma consistió en la explicitación en clase del guión experto por parte del docente, y su enseñanza a través de un proceso secuenciado. Todo el trabajo constituyó para el docente un aporte que éste valoró positivamente, y a su juicio no demandó esfuerzos mayores de planificación y no lo distrajo de sus tareas centrales en el aula. Lo más importante de todo es que piensa seguir utilizándolo. Al ser entrevistado al año siguiente de la intervención, sostuvo que cree que utiliza espontáneamente algunos aspectos de la metodología cuando presenta textos que considera de dificultad para los alumnos. Destacó especialmente que le resultó una estrategia útil para que los alumnos pierdan su miedo a la lectura.

Los principales límites del presente estudio, se vinculan al control de variables que puede realizarse en situaciones de aula. Las opciones metodológicas se orientaron a ganar en validez ecológica (Alfassi, 2004; Paris y Paris, 2001) en detrimento de un control de variables más fuerte. Por ejemplo, ambos grupos trabajaron con el mismo docente, registrando las clases de forma magnetofónica. Con el propósito de ganar en control podría utilizarse un segundo grupo de comparación a cargo de otro docente, realizando las evaluaciones correspondientes. También se podrían haber conocido más datos sobre la opinión de los estudiantes sobre el docente y haber contado con más información sobre las competencias psicológicas de los estudiantes. Pero el haber llevado a cabo estas averiguaciones hubiese supuesto una injerencia mayor en el transcurrir de la docencia y probablemente hubiese supuesto una merma en el efecto de la intervención al haberse hecho de forma mucho más artificiosa.

En cuanto a los instrumentos de evaluación, una dificultad que surgió fue diseñar cuestionarios de comprensión de textos comparables y ajustados a los contenidos de la asignatura. 
Fue difícil diseñar cuestionarios totalmente equiparables. Para otros trabajos futuros sería bueno considerar otras medidas de comprensión como la transferencia a textos de otras asignaturas, que nos permitirían ver en qué medida lo aprendido de autorregulación se generaliza.

La tarea de pensamiento en voz alta resultó muy interesante para evaluar la autorregulación del aprendizaje, aunque implicó gran dificultad para los alumnos que debieron realizarla. En estudios futuros, a los efectos de sacar más provecho aún de la tarea, podría resultar útil modelar la presentación de la consigna y entrenarlos previamente en la misma, tal como se ha realizado en otras investigaciones (Cerdán, 2007; Vidal-Abarca \& Cerdán, 2008).

En este caso, no se realizaron evaluaciones individuales de los sujetos del grupo control en la tarea de pensamiento en voz alta, lo cual sería muy interesante a la hora de determinar, aún con mayor rigurosidad, los efectos de una enseñanza explícita en los procesos de autorregulación frente a lo que se consigue de forma más implícita en los grupos sin esta intervención.

Este trabajo ha tenido unas dimensiones modestas, se ha llevado a cabo con un solo docente y en dos grupos de clase. A partir de nuestros resultados resultaría interesante extender la experiencia a más docente y ampliar la base de participantes de la investigación. También podría extenderse a otras asignaturas en forma coordinada y simultánea.

En definitiva, pretendíamos evaluar la incidencia de la enseñanza de autorregulación del aprendizaje en un contexto real de aula a través de una herramienta de sencilla aplicación, para que pudiese ser incorporada al trabajo de los docentes en el aula. Se han encontrado resultados al respecto satisfactorios en orden a poder generar estrategias docentes para enseñar a pensar y enseñar a comprender.

Cabe recordar que nuestra metodología ha beneficiado especialmente a los alumnos en mayor riesgo académico, que son los que más lo necesitan.

Los resultados obtenidos invitan a seguir avanzando en el diseño de herramientas educativas que contribuyan a que los estudiantes se conviertan en auténticos gestores de su propio aprendizaje.

\section{REFERENCIAS}

Alfassi, M. (2004). Reading to learn: effects of combined strategy instruction on high school students. The journal of educational research, 97, 4, 171-184.

Alonso-Tapia, J. \& Carriedo, N. (2002). El entrenamiento de la comprensión lectora. En Corral, A. (coord.). Psicología del aprendizaje de textos. Madrid: UNED.

Boekaerts, M., \& Corno, L. (2005). Self-Regulation in the Classroom: A Perspective on Assessment and Intervention. Applied Psychology: An International Review, 54(2), 199-231.

Braten, I. \& Samuelstuen, M. (2004). Does the influence of reading purpose on reports of strategic text processing depend on students' topic knowledge? Journal of educational psychology, 96, 2, 324-336.

Butler, D.; Beckingham, B. \& Novak, H. (2005). Promoting strategic learning by eighth-grade students struggling in mathematics: a report of three case studies. Learning disabilities research \& practice, 20, 3, 156174.

Cerdán, R. (2007). Estrategias de lectura y contestación a preguntas con textos múltiples: Un estudio de pensamiento en voz alta. Infancia y Aprendizaje, 30(1), 55-71.

Cerdán, R., \& E. Vidal-Abarca. (2008). The effects of tasks on integrating information from multiple documents. Journal of Educational Psychology, 100(1), 209-222.

Eme, E., Puustinen, M., \& Coutelet,B. (2006). Individual and developmental differences in reading monitoring: when and how do children evaluate their comprehension? European Journal of Psychology of Education, 21, 1, 91-115.

Fuchs, L.; Fuchs, D.; Prentice, K.; Burch, M.; Hamlett, C.; Owen, R. \& Schroeter, K. (2003). Enhancing third-grade students' mathematical problem solving with selfregulates learning strategies. Journal of educational psychology, 95, 2, 306-315.

Guasch, T., \& Castelló, M. (2002). Aproximación a la enseñanza de la toma de apuntes en la Educación Secundaria Obligatoria: un estudio descriptivo. Infancia y Aprendizaje, 25, 2, 169-181.

Kitsantas, A. (2002). Test preparation and performance: a self-regulatory análisis. The journal of experimental education, 70, 2, 101-113.

Mason, L. (2004). Explicit Self-Regulated strategy development versus reciprocal questioning: effects on expository reading comprehension among struggling readers. Journal of Educational Psychology, 96, 2, 283-296.

Mateos, M. (2001). Metacognición y educación. Buenos Aires: Aique.

Nietfeld, J.; L. Cao \& J. Osborne. (2005). Metacognitive monitoring accuracy and student performance in the postsecondary classroom. The journal of experimental education, 74, 1, 7-28.

Onwuegbuzie, A.; J. Slate \& R. Schwartz. (2001). Role of study skills in graduate-level educational research courses. The journal of educational research, 94, 4, 238-246. Paris, S. G., \& Paris, A. H. (2001). Classroom Applications of Research on Self-Regulated Learning. Educational Psychologist, 36(2), 89-101. 
Pintrich, P. (2003). A Motivational Science Perspective on the Role of Student Motivation in Learning and Teaching Contexts. Journal of Educational Psychology, 95(4), 667-686.

Pozo, J. I. (2008). Aprendices y maestros. La psicología cognitiva del aprendizaje. 2da Ed. Madrid: Alianza.

Roeschl-Heils, A.; Schneider, W., \& van Kraayenoord, C. (2003). Reading, metacognition and motivation: a follow-up study of german students in grades 7 and 8. European journal of psychology of education, 18, 1, 75-86.

Sungur, S. \& Tekkaya, C. (2006). Effects of problem-based learning and tradicional instruction on self-regulated learning. The journal of educational research, 99, 5, 307-317.
Thiede, K.; Anderson, M., \& Therriault, D. (2003). Accuracy of metacognitive monitoring affects learning of texts. Journal of educational psychology, 2003, 95, 1, 66-73.

Torrance, M.; Fidalgo, R., \& García, J. (2007). The teachability and effectiveness of cognitive self-regulation in sixth-grade writers. Learning and instruction, 17, 265285.

Zimmerman, B. (2000). Attaining self-regulation. A social cognitive perspective. En Boekaerts, M., P. Pintrich \& M. Zeidner (eds.) Handbook of self-regulation. New York: Academic Press.

Zimmerman, B. (2008). Investigating self-regulation and motivation: Historical background, methodological developments, and future prospects. American Educational Research Journal, 45(1), 166-183. 